Chem. Ber. 110, 3745 - 3758 (1977)

\title{
Einige Diels-Alder-Additionen des Benzvalens
}

\author{
Manfred Christl ${ }^{*}$,Hans-Joachim Lüddeke ${ }^{1)}$, Anton Nagyrevi-Neppel ${ }^{2)}$ und \\ Gertrud Freitag
}

Institut für Organische Chemie der Universität Würzburg,

Am Hubland, D-8700 Würzburg

Eingegangen am 31. Januar 1977

Zwei 1,2,4,5-Tetrazine, ein 4,5-Dihydropyridazin, zwei ortho-Benzochinone und Hexachlorcyclopentadien liefern als elektronenarme Diene glatt mit der elektronenreichen Doppelbindung des Benzvalens die erwarteten Addukte (5a,b, 7, 9a,b, 16a). Die Umwandlung von 9 b und 16a macht das neue $\mathrm{C}_{10} \mathrm{H}_{10}$-Isomere Tetracyclo[4.4.0.0.4.4. Isomere Pentacyclo[6.2.1. $\left.0^{2,7} .0^{3.5} .0^{4.6}\right]$ undec-9-en (16 b) bequem zugänglich. Die Konstitutionen der neuen Verbindungen werden anhand ihrer ${ }^{13} \mathrm{C}$-NMR-Spektren gesichert, die ein eindeutiges Kriterium für die Analyse derartiger, das Bicyclo[1.1.0]butan-System enthaltender Polycyclen bieten.

\section{Some Diels-Alder Additions of Benzvalene}

Being electron-deficient dienes, two 1,2,4,5-tetrazines, one 4,5-dihydropyridazine, two orthobenzoquinones and hexachlorocyclopentadiene add readily to the electron-rich double bond of benzvalene to form the expected products $(5 \mathrm{a}, \mathrm{b}, 7,9 \mathrm{a}, \mathrm{b}, 16 \mathrm{a})$. Transformation of $9 \mathrm{~b}$ and $16 \mathrm{a}$ renders the new $\mathrm{C}_{10} \mathrm{H}_{10}$ isomer tetracyclo $\left[4.4 .0 .0^{2,4} .0^{3,5}\right]$ deca-7,9-diene (11b) and the new $\mathrm{C}_{1,}, \mathrm{H}_{12}$ isomer pentacyclo[6.2.1.0 $\left.0^{2.7} \cdot 0^{3.5} \cdot 0^{4.6}\right]$ undec-9-ene (16b) respectively easily accessible. The structures of the new compounds are proved by their ${ }^{13} \mathrm{C}$ NMR spectra, which offer an unambiguous criterion for the analysis of such polycycles including the bicyclo[1.1.0]butane system.

Wie im Norbornen (1) ist auch die Doppelbindung im Benzvalen ${ }^{3 \prime}$ (2) Bestandteil eines gespannten Fünfrings und zu vielen Additionsrcaktionen fähig. Stufenweise verlaufende Anlagerungen elektrophiler Partner gehen bei 1 und 24) häufig mit Gerüstumlagerungen einher, während im Falle synchroner Additionen an die 2-Doppelbindung wie derjenigen von Wasserstoff mit $\mathrm{Di}$ imin $^{\text {5), von Dihalogencarbenen }}{ }^{6.7)}$, Dichlorketen ${ }^{8)}$, 1,3-Dipolen ${ }^{6)}$ und einem Tetrazin ${ }^{6)}$ das Bicyclo[1.1.0]butan-System erhalten bleibt. Die vorliegende Arbeit gibt erweiterte Auskunft über die Eignung von 2 als Dienophil bei Diels-Alder-Additionen.

\footnotetext{
1) Forschungspraktikant, Univ. München, März bis Mai 1973.

2) Forschungspraktikant, Univ. München. November 1973 bis Januar 1974

3) Leicht zugänglich nach: T. J. Katz, E. J. Wang und N. Acton, J. Am. Chem. Soc. 93, 3782 (1971);

T. J. Katz, R. J. Roth, N. Acton und E. J. Carnahan, Org. Synth. 53, 157 (1973).

4) R. J. Roth und T. J. Katz, J. Am. Chem. Soc. 94, 4770 (1972); T. J. Katz und N. Acton, ebenda 95, 2738 (1973); T. J. Katz und K. C. Nicolaou, ebenda 96, 1948 (1974).

5) M. Christl und G. Brüntrup, Chem. Ber. 107, 3908 (1974).

6) $M$. Christl, Angew. Chem. 85, 666 (1973); Angew. Chem., Int. Ed. Engl. 12, 660 (1973).

"M. Christl und G. Brüntrup, Angew. Chem. 86, 197 (1974); Angew. Chem., Int. Ed. Engl. 13, 208 (1974).

B) G. E. Gream, L. R. Smith und J. Meinwald, J. Org. Chem. 39, 4361 (1974); L. R. Smith, G. E. Gream und J. Meinwald, ebenda 42, 927 (1977).

(c) Verlag Chemie, GmbH, D-6940 Weinheim, 1977
} 


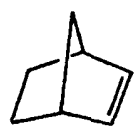

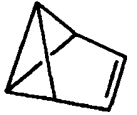

Da Benzvalen (2) in reinem Zustand detoniert ${ }^{3)}$ und in etherischer Lösung bei Raumtemperatur in einigen Tagen in Benzol ubbergeht, darf es bei Umsetzungen nicht erhitzt werden.

\section{A. 1,2,4,5-Tetrazine}

Sie sind bei Diels-Alder-Additionen ${ }^{9)}$ als Dienkomponenten besonders reaktiv. 3,6-Diphenyl- (3a) und 3,6-Bis(methoxycarbonyl)tetrazin (3b) liefern mit Norbornen (1) hohe Ausbeuten der erwarteten Produkte ${ }^{10,11}$.

Bei der Behandlung von 3a mit etherischer Benzvalenlösung (2) verschwanden unter Gasentwicklung die violetten Kristalle, und ein gelber Niederschlag fiel aus. Aufgrund der Elementaranalyse des mit 93\% Rohausbeute erhaltenen Produkts liegt die erwartete Bruttoformel von 5a vor. Das Dienophil 2 lagert sich an das Dien 3a unter Bildung des 1:1-Addukts $\mathbf{4} \mathbf{a}$, das in einer Diels-Alder-Cycloreversion ein Molekül Stickstof verliert und 5a hervorbringt. Die $c_{\mathbf{s}}$-Symmetrie offenbart sich im ${ }^{1} \mathrm{H}-\mathrm{NMR}$-Spektrum in den Signalen der aliphatischen Wasserstoffe. Von 1-, 6-H und 2-, 5-H stammen die Banden bei $\delta 3.28$ und 2.50, während 3-H und 4-H ein AB-System mit den chemischen Verschiebungen 2.23 und 1.88 sowie der Kopplung $J_{3,4}=9.7 \mathrm{~Hz}$ zeigen. In Úbereinstimmung mit den Verhältnissen am Bicyclo[1.1.0]butan ${ }^{12)}$ und am Tricyclo[3.1.0.0.6. hexan $^{\text {s) }}$ (18) fallen die weiteren Kopplungen klein aus und äußern sich in Linienverbreiterungen und angedeuteter Feinstruktur.

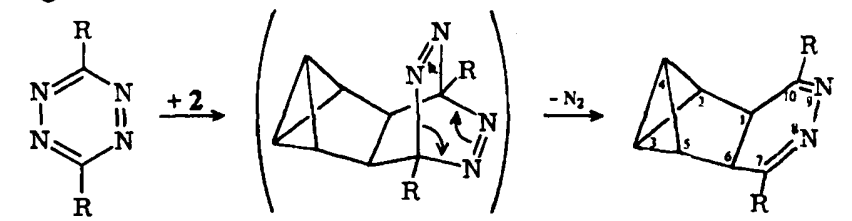

\begin{tabular}{l|lll}
$\mathrm{R}$ & & & \\
\hline $\mathrm{C}_{6} \mathrm{H}_{5}$ & $3 a$ & $4 a$ & $5 a$ \\
$\mathrm{CO}_{2} \mathrm{CH}_{3}$ & $3 b$ & $4 b$ & $5 b$
\end{tabular}

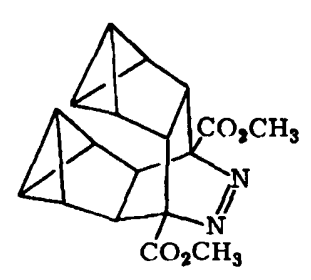

6

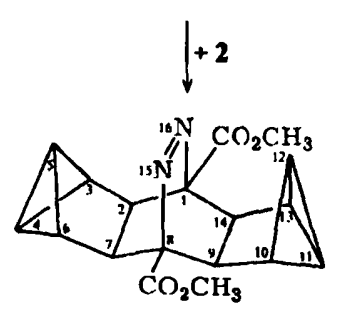

7

\footnotetext{
9) Aufsatz über präparative Aspekte: J. Sauer, Angew. Chem. 78, 233 (1966); Angew. Chem., Int. Ed. Engl. 5, 211 (1966).

10) J. Sauer und G. Heinrichs, Tetrahedron Lett. 1966, 4979.

11) W. Dittmar, G. Heinrichs, A. Steigel, T. Troll und J. Sauer, Tetrahedron Lett. 1970, 1623.

12) K. Wüthrich, S. Meiboom und L. C. Snyder, J. Chem. Phys. 52, 230 (1970).
} 
3 b ergab mit 2 in 87 proz. Rohausbeute den Tetracyclus 5 b, dessen ${ }^{1}$ H-NMR-Spektrum die Konstitution stützt. Als entscheidend erwies sich das $1: 1$-Verhältnis der Protonen der Methoxygruppen zu denen des Tricyclohexansystems. Für die $c_{s}$-Symmetrie spricht das Auftreten nur eines Methoxysinguletts $(\delta=4.05)$. Die weiteren Signale sind denen von 5a ähnlich.

Eine korrekte Elementaranalyse von $\mathbf{5 b}$ wurde nicht erhalten, was auf zwei Ursachen zurückgeht. Einmal war die Substanz nicht unverändert umkristallisierbar. Zum zweiten konnte zwecks quantitativer Umsetzung von 3b kein 2-Uberschuß verwendet werden, weil sich dieser in einer weiteren Diels-Alder-Addition mit 5b zu 7 vereinigte.

Präparativ gewann man den Octacyclus 7 durch Einwirkung von 2 auf 3 b in 69 proz. Ausbeute. Dabei entstand aus der zunächst roten Suspension rasch eine gelbe, in der 5b vorlag und deren Farbe im Verlauf von $20 \mathrm{~h}$ verblaßte. Das UV-Spektrum von 7 zeigt durch die Bande bei $\lambda=373 \mathrm{~nm}$ mit $\varepsilon=75$ den cis-Azochromophor an. Im ${ }^{1} \mathrm{H}$-NMRSpektrum verhalten sich die Absorptionen der Methoxygruppen zu denen der Tricyclohexansysteme erwartungsgemäß wie 1:2. Darüber hinaus signalisiert das Auftreten der Banden von nur fünff Protonensorten zwei Symmetrieebenen für das Molekül. Auch das ${ }^{13}$ C-NMR-Spektrum (Kap. E) steht im Einklang mit der vorgeschlagenen Konstitution.

Die analytischen Daten lassen aber eine Unterscheidung von der Alternativstruktur 6 nicht zu, da sie zwar eine andere Konfiguration aber die gleiche Symmetrie besitzt. Bezüglich des zentralen Cyclohexanrings in der Bootform sind die Tricyclohexansysteme in 7 exo,exo- und in 6 endo,endo-anelliert. Die weiterhin mögliche Konfiguration mit exo,endo-Anellierung scheidet aufgrund ihrer Symmetrieeigenschaften aus. Gegen 6 spricht sowohl die Aldersche endo-Regel ${ }^{13)}$ als auch die sterische Behinderung beider Kohlenwasserstoffreste, die bereits den Weg zu 6 energiereicher machen sollte. Bei der Norbornenaddition an $\mathbf{3 b}$ wurde anhand dort verfügbarer NMR-Argumente die zu 7 analoge Struktur gesichert ${ }^{11}$.

\section{B. ortho-Benzochinone}

Bei diesen Verbindungen können nach Horner und $M e r z^{14)}$ in Abhängigkeit von der Natur des Dienophils sowohl das Allkohlenstoffdiensystem des Sechsrings als auch das Heterodien der $\alpha$-Diketogruppierung als $4 \pi$-Elektronenkomponente wirken ${ }^{9.14}$.

Etherisches 2 reagierte mit Tetrachlor-o-chinon (8a) sofort unter Farbaufhellung, und man isolierte mit 90\% Ausbeute den Pentacyclus 9a. Bereits seine gelbe Farbe weist auf das $\alpha$-Diketostrukturelement hin. Im UV/VIS-Spektrum liegt die farbgebende Bande bei $\lambda=445 \mathrm{~nm}$ mit der charakteristisch niedrigen molaren Extinktion von 130, während starke Banden im IR-Spektrum bei 1742 und $1762 \mathrm{~cm}^{-1}$ ebenfalls für CO-Gruppen sprechen. Mit Ausnahme der Tatsache, daß nur Absorptionen aliphatischer Wasserstoffe vorhanden sind, enthält das ${ }^{1} \mathrm{H}$-NMR-Spektrum keine Informationen. Anders das ${ }^{13} \mathrm{C}$ NMR-Spektrum (Kap. E), das nicht nur die sieben Kohlenstofftypen anhand der Linienzahl charakterisiert, sondern diese schon aufgrund der chemischen Verschiebung in 13) Aufsatz uiber mechanistische Aspekte bei der Diels-Alder-Addition: J. Sauer, Angew. Chem.
79, 76 (1967); Angew. Chem., Int. Ed. Engl. 6, 16 (1967).

14) L. Horner und H. Merz, Liebigs Ann. Chem. 570, 89 (1950). 
Carbonyl-, Olefin-, chlorsubstituierte und unsubstituierte aliphatische Kohlenstoffe einteilt. Nimmt man die ${ }^{13} \mathrm{C}-\mathrm{H}-\mathrm{K}$ opplungskonstanten noch hinzu, so geben sich die Bicyclobutan-Brückenkopfkohlenstoffe $\mathrm{C}-4$ und $\mathrm{C}-5$ an den Werten von 224.8 und $223.5 \mathrm{~Hz}, \mathrm{C}-3,-6$ als Cyclopropankohlenstoffe an $173.9 \mathrm{~Hz}$ und C-2, -7 als Cyclopentankohlenstoffe an $145.8 \mathrm{~Hz}$ zu erkennen.

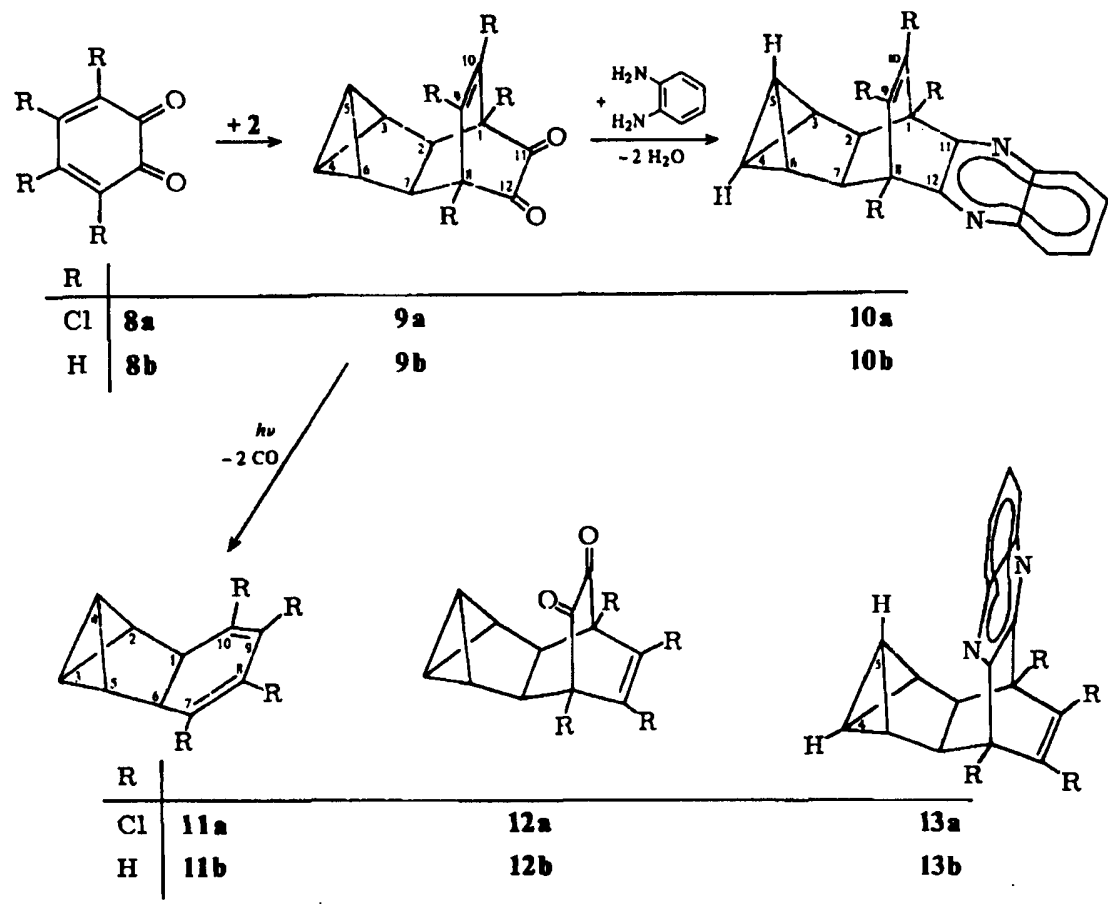

Die spektroskopischen Daten unterscheiden jedoch nicht zwischen 9a und seinem Isomeren 12a. Aus der leicht verlaufenden Chinoxalinbildung ${ }^{14)}$ der $\sigma$-Chinonaddukte mit $\alpha$-Diketogruppierung bei der Umsetzung mit o-Phenylendiamin leitet sich ein Kriterium her, das derartige Fragen zu lösen vermag ${ }^{15,16)}$, und mit dessen Hilfe auch die Strukturzuordnung der 1-Addukte von 8 a getroffen werden konnte ${ }^{16)}$. Angewandt auf das 2-Addukt besagt dies, daß im Chinoxalin 10a der Ringstrom des kondensierten Aromaten die NMR-Absorption von 4-H und 5-H praktisch nicht beeinflussen sollte. In 13a dagegen befindet sich 5- $\mathrm{H}$ im Bereich verstärkter Abschirmung durch den Aromaten, was mit einer erheblichen Hochfeldverschiebung seiner Absorption einhergehen sollte. Das Chinoxalinderivat wurde aus dem 2-Addukt mit $80 \%$ Ausbeute dargestellt. Sein NMR-Spektrum zeigt die Absorptionen von 4-H und 5-H als Multiplett bei $\delta=$ 2.0-2.6, die damit an der gleichen Stelle erscheinen wie die des Ausgangsdiketons. Für das vorliegende 2-Addukt und sein Chinoxalinderivat können daher die Konstitutionen 9a bzw. 10a als bewiesen gelten.

15) E. E. Nunn, W. S. Wilson und R. N. Warrener, Tetrahedron Lett. 1972, 175

16) W. Friedrichsen und R. Epbinder, Tetrahedron Lett. 1973, 2059. 
Im Gegensatz zu den zahlreichen Umsetzungen substituierter $o$-Chinone, besonders des Tetrachlorderivats 8a, sind erfolgreiche Diels-Alder-Additionen der unsubstituierten Stammverbindung $\mathbf{8 b}$, in denen sich diese als Dien verhält, recht selten. Obwohl gemäß der NMR-Kontrolle die Anlagerung von 2 an $\mathbf{8 b}$ deutlich langsamer verlief als an $\mathbf{8 a}$, isolierte man nach $18 \mathrm{~h}$ bei Raumtemperatur doch $58 \%$ des Addukts $9 \mathrm{~b}$. In seinem ${ }^{1} \mathrm{H}$ NMR-Spektrum gesellen sich jetzt zu den Signalen des Tricyclohexansystems auch die Banden von 1-, 8-H und 9-, 10-H als Multipletts bei $\delta=3.47$ bzw. 6.12. Das ${ }^{13} \mathrm{C}-\mathrm{NMR}$ Spektrum (Abb. 1) beseitigt letzte Zweifel an der Richtigkeit der Konstitution. Man beachte die für die Zuordnung entscheidenden Feinstrukturunterschiede (vgl. Kap. E). Die Konfigurationszuweisung beruht wieder auf dem ${ }^{1} \mathrm{H}-\mathrm{NMR}$-Spektrum des zugehörigen Chinoxalins $10 \mathrm{~b}$, in dem die chemischen Verschiebungen von 4-H und 5-H praktisch nicht von den entsprechenden Werten in $9 \mathrm{~b}$ abweichen. 12b und $13 \mathrm{~b}$ scheiden daher als Alternativen aus, was die Gültigkeit der Alderschen endo-Regel ${ }^{13)}$ unterstreicht.

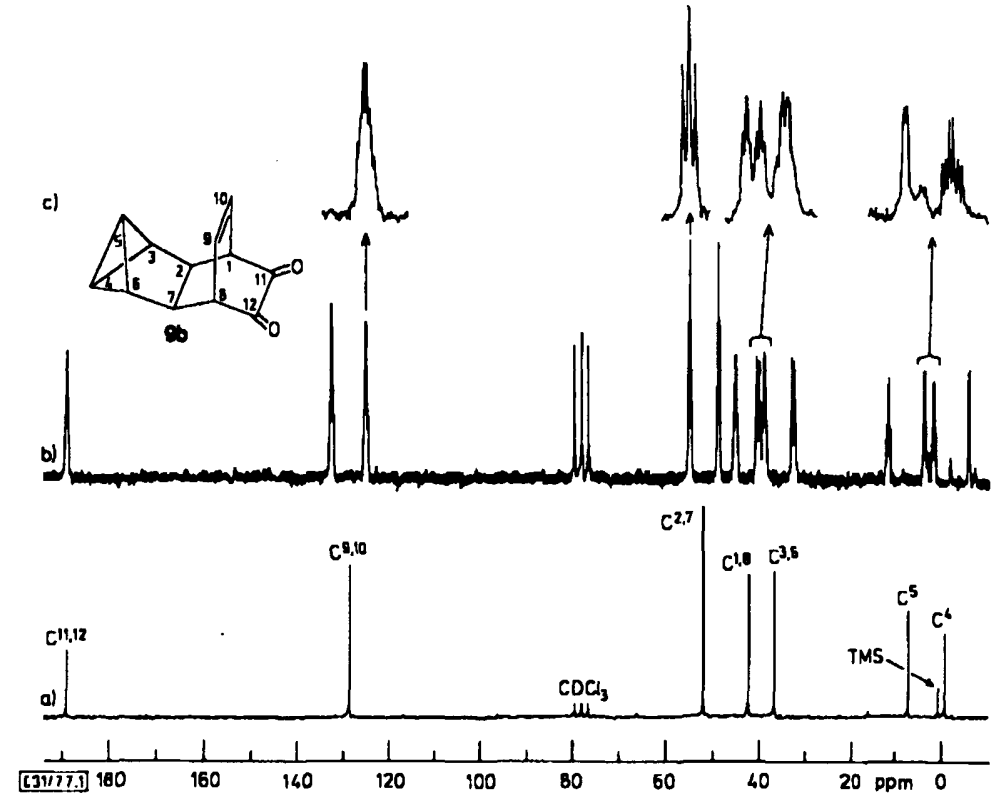

Abb. $1 .{ }^{13} \mathrm{C}$-NMR-Spektrum von Pentacyclo[6.2.2.0 2.7. $\left.0^{3.5} \cdot 0^{4.6}\right]$ dodeca-9-en-11,12-dion (9 b) bei $22.64 \mathrm{MHz}$ in $\mathrm{CDCl}_{3}$ :a) Breitbandentkoppelt, b) protonengekoppelt, c) auf das Fünffache gedehnte Ausschnitte aus b)

Die Frage, ob neben 9a und $9 \mathrm{~b}$ in geringer Menge auch $12 \mathrm{a}$ und $12 \mathrm{~b}$ sowie Benzodioxenderivate entstehen, zu deren Bildung die $\alpha$-Diketogruppierung als Dien zu fungieren hätte, wurde nicht untersucht.

Diels-Alder-Addukte von 0 -Chinonen mit $\alpha$-Diketostruktur spalten beim Belichten zwei Moleküle Kohlenmonoxid $\mathrm{ab}^{16-18}$. Die Anwendung dieser Reaktion überfürte 9a und 9 b mit 99 bzw. 37\% Ausbeute in die Tetracyclen 11a und 11 b. 11a weist sich

17) D. Bryce-Smith und A. Gilbert, Chem. Commun. 1968, 1702.

18) J. Strating, B. Zwanenburg, A. Wagenaar und A. C. Udding, Tetrahedron Lett. 1969, 125. 
durch sein ${ }^{1} \mathrm{H}$-NMR-Spektrum, -das denen der verwandten Heterocyclen 52 und $5 \mathrm{~b}$ ähnlich ist, als Derivat des Tricyclohexans 18 aus. Neben den schmalen Multipletts für 1-, 6-H und 2-, 5-H zeigen 3-H und 4-H das erwartete AB-Spektrum mit $J_{3,4}=9.0 \mathrm{~Hz}$, in dem jede Linie durch die Kopplungen mit 2-, 5- $\mathrm{H}(1.6$ und $1.9 \mathrm{~Hz})$ und 1-, 6- $\mathrm{H}(0.75$ und $1.15 \mathrm{~Hz}$ ) zum Tripeltriplett aufgespalten ist. Weniger informativ ist das ${ }^{2} \mathrm{H}-\mathrm{NMR}$ Spektrum von $11 \mathrm{~b}$. Sowohl in $\mathrm{CDCl}_{3}$ als auch in $\mathrm{C}_{6} \mathrm{D}_{6}$ treten nur vier etwas verbreiterte Singuletts mit angedeuteter Feinstruktur auf, die über das korrekte Verhältnis von olefinischen zu aliphatischen Protonen hinaus keine weiteren Parameter preisgeben. Im Gegensatz dazu erbringt das ${ }^{13} \mathrm{C}$-NMR-Spektrum von $11 \mathrm{~b}$ (Abb. 2), wie auch das von $11 \mathrm{a}$, anhand von Linienzahl, chemischen Verschiebungen und ${ }^{13} \mathrm{C}-\mathrm{H}$-Kopplungskonstanten den eindeutigen Strukturbeweis (Kap. E).

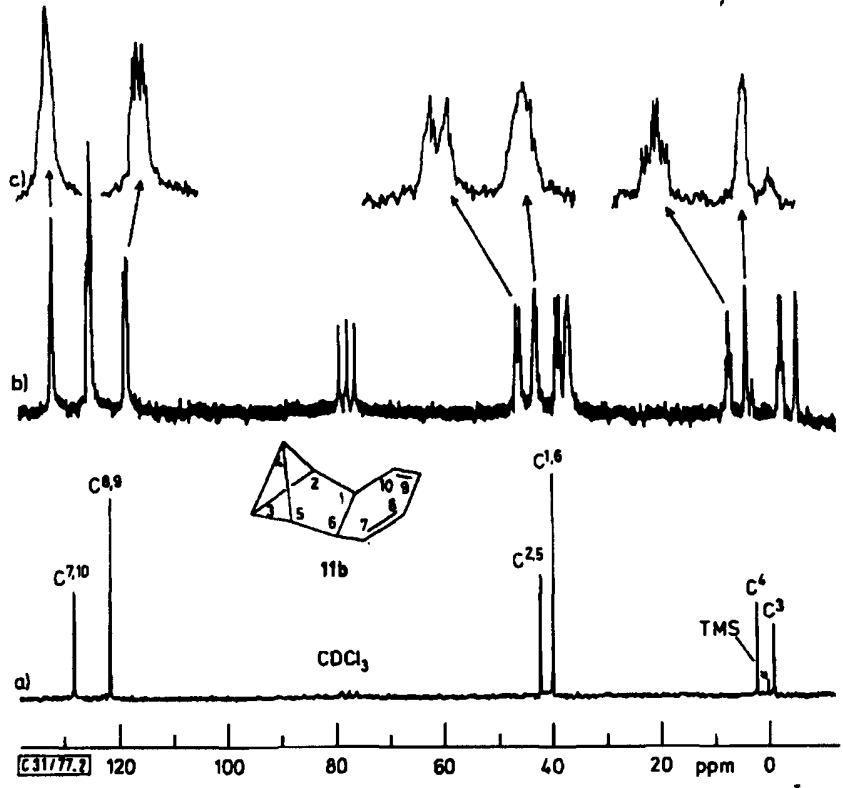

Abb. $2 .{ }^{13} \mathrm{C}$-NMR-Spektrum von Tetracyclo[4.4.0.02.4. $0^{3,5}$ ]deca-7,9-dien (11 b) bei $22.64 \mathrm{MHz}$ in $\mathrm{CDCl}_{3}$ : a) Breitbandentkoppelt, b) protonengekoppelt, c) auf das Fünffache gedehnte Ausschnitte aus b)

Tetracyclo[4.4.0.0 2,4.0 $0^{3,5}$ ]deca-7,9-dien (11b) ist ein neues Mitglied der interessanten Familie der $\mathrm{C}_{10} \mathrm{H}_{10}$-Kohlenwasserstoffe. Es kann, wie hier beschrieben, ebenso wie sein Tetrachlorderivat 11a aus Benzvalen (2) bequem in zwei Schritten dargestellt werden. Kürzlich sind zwei hochsubstituierte Derivate von 11b bekannt geworden, nämlich das 1,6-Bis(methoxycarbonyl)2,3,4,5-tetramethyl-Derivat ${ }^{19)}$ und das 1,2,3,4,5,6-Hexakis(trifluormethyl)-Derivat. Letzteres wurde von zwei Arbeitsgruppen ausgehend vom Per(trifluormethyl)benzvalen über das Butadien-20) bzw. Cyclobutadien-Addukt ${ }^{21)}$ bereitet. Das Ringsystem von 5a und b stellt ein Diazaderivat von 11 b dar, von dem auch der Octamethylabkömmling ${ }^{19)}$ schon beschrieben ist.

19) G. Maier, Angew. Chem. 86, 491 (1974); Angew. Chem., Int. Ed. Engl. 13, 425 (1974).

20) Y. Kobayashi, I. Kumadaki, A. Ohsawa, Y. Hanzawa und M. Honda, Tetrahedron Lett. 1975, 3819.

21) R. N. Warrener, E. E. Nunn und M. N. Paddon-Row, Tetrahedron Lett. 1976, 2639. 


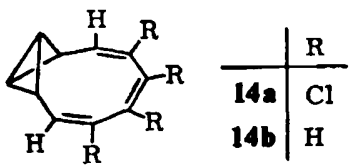

Als 1,3-Cyclohexadiene sollten $11 \mathrm{a}$ und b fahig sein, in thermisch erlaubter Reaktion die Sechsringe disrotatorisch zu den Hexatrienderivaten 14a bzw. b zu öfnen. Einen Hinweis auf die Existenz einer solchen Valenztautomerie erwartete man von der Temperaturabhängigkeit des ${ }^{\mathbf{}} \mathbf{H}$ NMR-Spektrums von 11a. Die Signale von 3-H und 4-H sollten koaleszieren, falls 14a auftritt, da über diese $\mathrm{Zwischenstufe} \mathrm{3-H}$ und 4-H ihre Identität austauschen könnten. Tatsächlich veränderte sich jedoch das ' $\mathrm{H}$-NMR-Spektrum von $11 \mathrm{a}$ in Diphenylether bis $200^{\circ} \mathrm{C}$ nicht, so daß wenigstens bis zu dieser Temperatur besagte elektrocyclische Ringöfnung nicht mit nennenswerter Geschwindigkeit stattfindet.

\section{Hexachlorcyclopentadien}

Unter den zahlreichen Olefinen, die mit diesem Dien (15) umgesetzt wurden ${ }^{22)}$, befindet sich auch Norbornen (1), das bei Temperaturen über $100^{\circ} \mathrm{C}$ in guten Ausbeuten ein 1:1-Addukt liefert. Benzvalen (2) dagegen erbrachte schon bei Raumtemperatur nach $22 \mathrm{~h}$ in 72 proz. Ausbeute das Produkt 16a. Sein ${ }^{1} \mathrm{H}-\mathrm{NMR}-\mathrm{Spektrum}$ weist nur zwei breite Banden im Bereich aliphatischer Protonen auf. Für das endo-Isomere 16a spricht im Gegensatz zur exo-Verbindung 17a die Aldersche endo-Regel ${ }^{131}$.<smiles>ClC1=C(Cl)C(Cl)(Cl)C(Cl)=C1Cl</smiles>

15

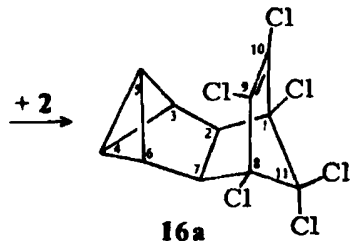

Na,

169

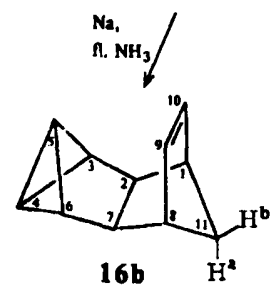

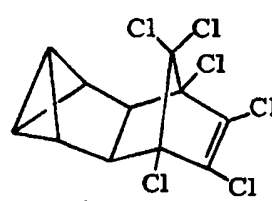

172

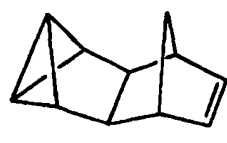

$17 \mathrm{~b}$

Die reduktive Dechlorierung zum unsubstituierten Kohlenwasserstoff, der sich nicht direkt aus Cyclopentadien und 2 darstellen ließ, gelang in einem Schritt mit Natrium in flüssigem Ammoniak mit 31\% Ausbeute. Unter der Annahme, daß sich bei dieser Reaktion die Konfiguration nicht ändert, sollte $16 \mathrm{~b}$ - ein neues $\mathrm{C}_{11} \mathrm{H}_{12}$-Isomeres das Produkt sein. Das ${ }^{1} \mathrm{H}-\mathrm{NMR}$-Spektrum gibt das Verhältnis der Zahl der olefinischen zur Zahl der aliphatischen Protonen korrekt wieder. Überdies lassen Entkopplungsexperimente im gut aufgelösten $90-\mathrm{MHz}$-Spektrum die genaue Zuordnung einiger Parameter zu, so z.B. diejenigen des zur Doppelbindung anti-ständigen $11 \mathrm{a}-\mathrm{H}$ wegen

22) Zusammenfassung über Reaktionen des Hexachlorcyclopentadiens: $H$. E. Ungnade und E. T. Mc Bee, Chem. Rev. 58, 249 (1958). 
seiner charakteristischen Long-range-Kopplung ${ }^{23)} \mathrm{zu}$ 9-, 10-H. Einstrahlung bei $\delta=5.89$ überführt das Doppeltripeltriplett bei 1.36 in ein Doppeltriplett mit der 1.6-Hz-Kopplung zu 1-, 8-H, die gelöscht wird, wenn man bei 2.59 entkoppelt. Mit Hilfe des ${ }^{13} \mathrm{C}-\mathrm{NMR}$ Spektrums (Kap. E) läßt sich tatsächlich nicht nur die Gesamtstruktur 16b untermauern, sondern auch ihre Natur als endo,endo'-disubstituiertes Norbornen gegenüber der exo,exo'-Alternative 17b wahrscheinlich machen.

\section{Diskussion der Reaktivität des Benzvalens}

Obwohl kinetische Messungen ausstehen, läßt sich qualitativ feststellen, daß Benzvalen (2) in den erfolgreichen Umsetzungen mindestens gleich schnell reagiert wie Norbornen (1), eher noch etwas schneller. Beide Olefine enthalten eine dialkylierte CCDoppelbindung und können daher als elektronenreich gelten. Deshalb ist es kein Zufall, $\mathrm{da} B$ die verwendeten Diene ausnahmslos elektronenarm sind, die oben beschriebenen Diels-Alder-Additionen also unter die Kategorie derer mit inversem Elektronenbedarf ${ }^{131}$ fallen.

Als konzertierte $\left[{ }_{n} 4,+{ }_{n} 2\right]$-Cycloaddition ${ }^{24)}$ unterliegt die Diels-Alder-Reaktion der Orbitalkontrolle, die durch die HOMO-LUMO-Wechselwirkung zum Ausdruck gebracht werden kann. Bei obigem Reaktionstyp mit inversem Elektronenbedarf bestimmt die Beziehung zwischen dem LUMO des Diens und dem HOMO des Dienophils die Reaktivität ${ }^{25}$. Gleiches Dien vorausgesetzt, heißt dies, daß jenes Dienophil schneller addiert, welches das energiereichere HOMO besitzt, weil letzteres näher an das DienLUMO heranrückt und so die Wechselwirkung verbessert. Das Ionisationspotential des Benzvalens, das als Maß für die Lage des HOMOs gilt, wurde kürzlich photoelektronenspektroskopisch zu $8.55 \mathrm{eV}$ bestimmt ${ }^{26)}$. Es ist also um $0.42 \mathrm{eV}$ kleiner als das des Norbornens $(8.97 \mathrm{eV})^{27)}$. Dies läßt in Ubereinstimmung mit obigen qualitativen Befunden vermuten, daß bei Umsetzungen mit elektronenarmen Reaktionspartnern das Benzvalen dem Norbornen überlegen ist.

\section{E. ${ }^{13}$ C-NMR-Spektren}

Im Schema 1 sind ${ }^{13} \mathrm{C}$-chemische Verschiebungen und die über eine Bindung reichenden

${ }^{13} \mathrm{C}-\mathrm{H}$-Kopplungskonstanten einiger der oben besprochenen Verbindungen und dazugehöriger Modellsubstanzen angegeben. Die durch Diels-Alder-Additionen an das Benzvalen erhaltenen Verbindungen besitzen alle noch das Tricyclo[3.1.0.0 2.6 $]$ hexangerüst, was den Vergleich mit den Daten der Stammverbindung $18^{\text {5) }}$ rechtfertigt. Den Positionen 1 und 6 in 18 entsprechende Kohlenstoffe in 7,9a, 9b, 11a, 11 b und 16 beichen sowohl mit ihren chemischen Verschiebungen als auch mit ihren Kopplungskonstanten nur wenig von den Werten in 18 selbst ab. Damit bestätigt sich das Ergebnis einer kürzlich publizierten ${ }^{13} \mathrm{C}$-NMR-Studie ${ }^{28}$ ) über Bicyclo[n.1.0]-Kohlenwasserstoffe, daß Bi-

\footnotetext{
23) Vgl. E. I. Snyder und B. Franzus, J. Am. Chem. Soc. 86, 1166 (1964).

24) R. B. Woodward und R. Hoffmann, Angew. Chem. 81, 797 (1969); Angew. Chem., Int. Ed. Engl. 8, 781 (1969).

25) $R$. Sustmann, Tetrahedron Lett. 1971, 2721.

26) P. Bischof, R. Gleiter und E. Müller, Tetrahedron 32, 2769 (1976); P. J. Harman, J. E. Kent, T. H. Gan, T. B. Peel und G. D. Willet, J. Am. Chem. Soc. 99, 943 (1977).

27) P. Bischof, J. A. Hashmall, E. Heilbronner und V. Hornung, Helv. Chim. Acta 52, 1745 (1969).

28) M. Christl, Chem. Ber. 108, 2781 (1975).
} 
cyclo[1.1.0]butane anhand dieser besonders charakteristischen Parameter der Brückenkopfkohlenstoffe eindeutig identifiziert werden können. Auch die zu C-2, -5 in 18 analogen Kohlenstoffe in den polycyclischen Derivaten weisen recht konstante chemische Verschiebungen von 34.9-42.9 ppm und für Cyclopropankohlenstoffe typische Kopplungskonstanten von $162-174 \mathrm{~Hz}$ auf.

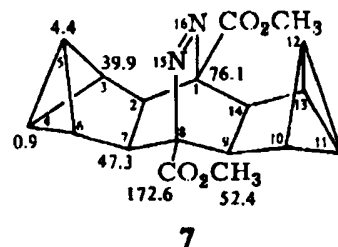

7

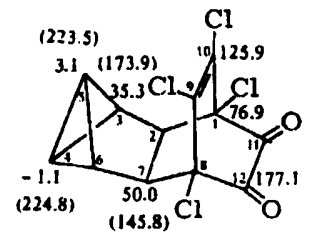

9a

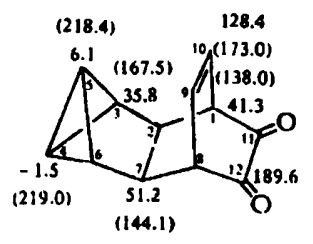

$9 \mathbf{b}$

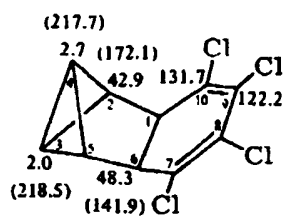

11 a

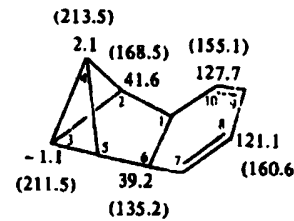

$11 \mathrm{~b}$

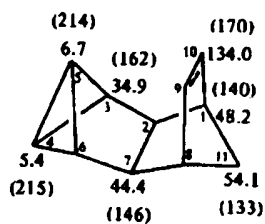

$16 b$

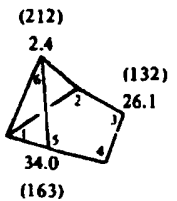

$18^{5)}$

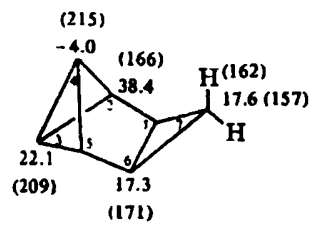

1928)

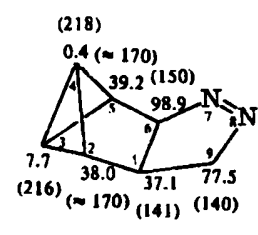

$20^{28}$

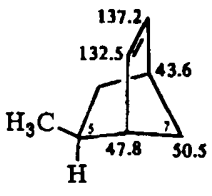

$21^{31)}$

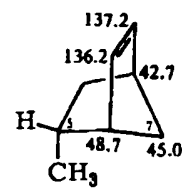

$22^{31)}$

Schema 1. ${ }^{13} \mathrm{C}$-Chemische Verschiebungen (in ppm feldabwärts von Tetramethylsilan) und ${ }^{13} \mathrm{C}-\mathrm{H}-\mathrm{K}$ opplungskonstanten (in Hertz) von einigen polycyclischen, das Tricyclo[3.1.0.0 $\left.0^{2,6}\right]$ hexan-Gerüst enthaltenden Systemen und Vergleichssubstanzen

Wegen der X̊hnlichkeit ihrer chemischen Verschiebungen erschien es zunächst nicht möglich, C-3 und C-4 in 11a und 11 b sowie C-4 und C-5 in 9a, 9b und $16 \mathrm{~b}$ spezifisch zuzuordnen. Jedoch ergab sich anhand der Feinstruktur im protonengekoppelten Spektrum ein eindeutiges Unterscheidungskriterium. Im Tetracyclo $\left[4.1 .0 .0^{2.4} .0^{3.5}\right]$ heptan (19) zeigen C-3 und C-4 wegen des richtungsabhängigen Effekts des 1,6-anellierten Cyclopropanrings eine Differenz ihrer chemischen Verschiebungen von $26.1 \mathrm{ppm}$, was die sichere Zuordnung gestattet ${ }^{28}$ ). Das protonengekoppelte Spektrum von ${ }^{19}{ }^{28}$ ) weist darüber hinaus fir die Signale von C-3 und C-4 unterschiedliche Feinstrukturen auf. Die Triplettaufspaltung bei C-3 deutet auf zwei Long-range-Kopplungen mit je $4.1 \mathrm{~Hz}$, von denen eine durch Vergleich mit 7-Halogenderivaten von $19^{29)} 7-e x o-H$ und die andere

29) $M$. Christl, unveröffentlichte Ergebnisse. 
4-H zugeschrieben werden muß. C-4 dagegen verfügt über mehrere weitreichende Kopplungen, wie an den breiteren, weniger gut aufgelösten Signalen erkennbar ist ${ }^{28}$. Mit Vergrößerung des 1,6-anellierten Rings schrumpft die C-3/C-4-Verschiebungsdifferenz zusammen, bei 20 beträgt sie nur noch $7.3 \mathrm{ppm}^{28}$. Im protonengekoppelten Spektrum von 20 findet sich jedoch noch die gleiche Feinstrukturcharakteristik wie bei 19: Die schmalen Linien des 216-Hz-Dubletts von C-3 verbergen nur geringfügige Long-rangeKopplungen, während das 218-Hz-Dublett von C-4 noch die Feinstruktur eines Doppeltripletts mit einer Kopplungskonstante von ca. $4 \mathrm{~Hz}$ und zwei gleichen Kopplungskonstanten von ca. $8 \mathrm{~Hz}$ beinhaltet. Genau dieses Aufspaltungsmuster findet man nun für die Bicyclobutan-Brückenkopikohlenstoffe $C-3$ und $C-4$ in 11a und b sowie $C-4$ und $C-5$ in $9 a, b$ und 16b. Man darf daher als gesichert ansehen, daB jeweils der bezüglich des 1,6- bzw. 2,7-anellierten Systems anti-ständige Kohlenstoff - C-3 in 11 a und b bzw. C-4 in 9a, b und $16 \mathbf{b}$ - die kleineren, nicht aufgelösten Long-range-Kopplungen tätigt, also die schmalen Signale besitzt. In den Abb. 1 und 2 ist dies an den Beispielen $9 \mathrm{~b}$ und $11 \mathrm{~b}$ demonstriert.

Daraus leitet sich für 9a, b, 11a, b und 16b die interessante Konsequenz $a b$, daß von den beiden Bicyclobutan-Brückenkopfkohlenstoffen nicht mehr derjenige bei höherem Feld absorbiert, der syn-ständig zum 1,6-bzw. 2,7-anellierten System fixiert ist, der sterisch bedingte $\gamma$-Effekt hier also keine Zuordnungshilfe mehr bietet. In allen Fällen enthält das anellierte Gerüst einen Sechsring, so $\mathrm{da} B$ ein richtungsabhängiger elektronischer Effekt wie bei 19 und möglicherweise $20^{28}$ ) bereits entfallen dürfte. Andererseits könnte der normale $\gamma$-Effekt von $C-7,-10$ auf $C-4$ in 11 a und $b$ bzw. von $C-1,-8$ auf $C-5$ in 9 a,b und 16b durch sterisch bedingte gegenläufige $\delta$-Substituenteneinflüsse ${ }^{30)}$ von $C-8,-9$ bzw. C-9, -10 überdeckt werden. Obwohl von 7 ein protonengekoppeltes Spektrum nicht vorliegt, darf in Analogie zu den ähnlichen Systemen 9a und b das Bicyclobutan-Brückenkopfkohlenstoff-Signal bei höherem Feld den anti-ständigen C-4, -11 zugewiesen werden.

In Úbereinstimmung mit der zweifachen $c_{s}$-Symmetrie zeigt das Spektrum von 7 sieben Linien, unter denen die vier der beiden Tricyclohexansysteme und die zwei der Methoxycarbonylgruppen eine typische Lage einnehmen. Für C-1, -8 bewirkt die Azogruppierung als $\alpha$-Substituent eine Tieffeldverschiebung des Signals nach $76.1 \mathrm{ppm}$. 9a, b und 16b besitzen nur je eine Symmetrieebene, so daB die sieben Linien in jedem Spektrum der Erwartung entsprechen. Die Zuordnung von C-3,-6 gelingt eindeutig aufgrund der charakteristischen Cyclopropan- ${ }^{13} \mathrm{C}-\mathrm{H}-$ Kopplungskonstante. Zur Unterscheidung von $\mathrm{C}-2,-7$ und $\mathrm{C}-1,-8$ eignet sich die Feinstruktur dieser Banden. In 92 zeigt C-2, -7 , wegen der Chloratome an C-1, -8 nicht verwechselbar, neben der großen Aufspaltung durch die Kopplung über eine Bindung noch ein Triplett mit einem Linienabstand von $5.2 \mathrm{~Hz}$. Ein solches Muster mit Triplettlinienabstand $6.2 \mathrm{~Hz}$ beobachtet man im Spektrum von $9 \mathrm{~b}$ (Abb. 1) an der Bande bei $51.2 \mathrm{ppm}$, während das Signal mit der weniger deutlichen, eher zu einem Quadruplett tendierenden Feinstruktur bei $41.3 \mathrm{ppm}$ C-1, -8 zugeordnet werden muß. Die Verhältnisse in $16 \mathrm{~b}$ liegen ähnlich, jedoch ist wegen der schiechteren Qualität des Spektrums die Feinstruktur weniger deutlich.

30) S. H. Grover, J. P. Guthrie, J. B. Stothers und C. T. Tan, J. Magn. Reson. 10, 227 (1973); J. G. Batchelor, ebenda 18, 212 (1975). 
Die auf chemischem Wege nicht geklärte Frage der exo- oder endo-Anellierung des Tricyclohexan- an das Norbornensystem in $16 \mathrm{~b}$ läBt sich durch Vergleich mit den ${ }^{13} \mathrm{C}$ NMR-Spektren von 5-endo- und 5-exo-Methylnorbornen (21 und 22) lösen. Die exoMethylgruppe in 22 verschiebt die Resonanz von C-7 gegenüber Norbornen um 3.8 ppm nach höherem, die Resonanzen der olefinischen Kohlenstoffe C-2 und C-3 um 0.7 bzw. $1.7 \mathrm{ppm}$ nach tieferem Feld ${ }^{311}$. Die Signale des endo-Isomeren 21 offenbaren gegenläufige Effekte $(\Delta \delta \mathrm{C}-3=-3.0, \mathrm{C}-7=1.7 \mathrm{ppm})$, wobei $\mathrm{C}-2 \mathrm{mit} \Delta \delta=1.7 \mathrm{ppm}$ wohl wegen des $\delta$-Effekts der Methylgruppe ${ }^{30)}$ aus der Reihe tanzt ${ }^{31}$. Verglichen mit Norbornen erscheint nun in 16b das Signal der Methylenbrücke (C-11) um 5.3 ppm bei tieferem und das der Vinylenbrücke um $1.5 \mathrm{ppm}$ bei höherem Feld, womit seine Natur als endo,endo'disubstituiertes Norbornen naheliegt.

Die olefinischen Kohlenstoffsignale in $11 \mathrm{a}$ und $\mathrm{b}$ können nur versuchsweise zugeordnet werden. Als auffallend klein erweist sich der Bruttosubstituenteneffekt aller vier Chloratome, lediglich das Signal von C-1, -6 rückt um 9.1 ppm nach tieferem Feld. Beim Vergleich von 9 a mit 9 b zeigt sich an den Absorptionen der olefinischen Kohlenstoffe C-9,-10 mit $-2.5 \mathrm{ppm}$ ebenfalls nur ein kleiner Substituenteneffekt, dagegen wird die Bande von C-1, -8 um $35.6 \mathrm{ppm}$ nach tieferem Feld verschoben. An den Carbonylkohlenstoffsignalen beobachtet man einen Feldaufwärtseffekt um $12.5 \mathrm{ppm}$. Der gleichzeitige Ersatz von jeweils vier Wasserstoffen durch Chlor läßt bezüglich des Substituenteneffekts eines Chloratoms keine Aussage zu.

Die ${ }^{13} \mathrm{C}-\mathrm{H}$-Kopplungskonstanten über eine Bindung sind fast überall größer als in der Muttersubstanz 18, jedoch fält bei den unsubstituierten Kohlenwasserstoffen 16b und $11 \mathrm{~b}$ die Erhöhung recht klein aus. Größere Effekte in 9a, b und 11a gehen wohl auf die Heterosubstituenten zurück. Folgende Long-range- ${ }^{13} \mathrm{C}-\mathrm{H}-\mathrm{Kopplungskonstanten}$ können mit einiger Sicherheit zugeordnet werden: 9a: ${ }^{2} J_{\mathrm{C}-5,4-\mathrm{H}}=3.3 \mathrm{~Hz},{ }^{2} J_{\mathrm{C}-5,3(6)-\mathrm{H}}=$ $7.5 \mathrm{~Hz} ; 9 \mathrm{b:}{ }^{2} J_{\mathrm{C}-5,4-\mathrm{H}}=3.8 \mathrm{~Hz},{ }^{2} J_{\mathrm{C}-5,3(6)-\mathrm{H}}=7.8 \mathrm{~Hz} ; 20:{ }^{2} J_{\mathrm{C}-4,3-\mathrm{H}} \approx 4 \mathrm{~Hz},{ }^{2} J_{\mathrm{C}-4,2(5)-\mathrm{H}} \approx$

$8 \mathrm{~Hz} ; 11 \mathrm{a}:{ }^{2} J_{\mathrm{C}-4,3-\mathrm{H}}=4.0 \mathrm{~Hz},{ }^{2} J_{\mathrm{C}-4,2(5)-\mathrm{H}}=8.0 \mathrm{~Hz} ; 11 \mathrm{~b}:{ }^{2} J_{\mathrm{C}-4,3-\mathrm{H}}=4.0 \mathrm{~Hz},{ }^{2} J_{\mathrm{C}-4,2(5)-\mathrm{H}}$ $=8.5 \mathrm{~Hz}$.

Wir danken Herrn Dr. W. Buchner für die Aufnahme der ${ }^{13} \mathrm{C}-\mathrm{NMR}-\mathrm{Spektren}$ und der Deutschen Forschungsgemeinschaft für die freundliche Förderung.

\section{Experimenteller Teil}

Die ${ }^{13}$ C-NMR-Spektren wurden mit dem Puls-Fourier-Transformverfahren an einem Bruker WH-90-Gerät aufgenommen. Als Referenzsignal diente entweder die Absorption von internem Tetramethylsilan oder ein Lösungsmittelsignal, von dem aus dann auf die TMS-Skala umgerechnet wurde. Die Reproduzierbarkeit der ${ }^{13} \mathrm{C}$-chemischen Verschiebungen beträgt $0.1 \mathrm{ppm}$, während die Kopplungskonstanten je nach der Angabe im Schema 1 entweder auf $1 \mathrm{~Hz}$ oder $0.1 \mathrm{~Hz}$ genau sein dürften.

7,10-Diphenyl-8,9-diazatetracyclo/4.4.0.0.0.4. $0^{3,5} /$ deca-7,9-dien (5a): $500 \mathrm{mg}$ (2.13 mmol) 3,6-Diphenyl-1,2,4,5-tetrazin ${ }^{32}$ - (3a) wurden bei Raumtemp. mit $10 \mathrm{ml}$ etherischem Benzvalen ${ }^{31}$ (2) (3.70 mmol) versetzt und $\mathrm{th}$ gerührt. Dabei lösten sich die violetten Kristalle unter Gasentwicklung

31) G. C. Levy und G. L. Nelson, Carbon-13 Nuclear Magnetic Resonance for Organic Chemists, S. 61, Wiley-Interscience, New York, London, Sidney, Toronto 1972.

32) Das Dihydrotetrazin wurde nach M. O. Abdehl-Rahman, M. A. Kira und M. N. Tolba, Tetrahedron Lett. 1968, 3871, dargestcllt und gemä $B$ Lit. ${ }^{3.1}$ dehydriert.

33) J. Sauer, A. Mielert, D. Lung und $D$. Peter, (.hem. Ber. 98. 1435 (1965) 
allmählich auf, und ein gelber Niederschlag fiel aus, der abgesaugt und mit Ether gewaschen wurde: $570 \mathrm{mg}(93 \%)$ 5a mit Schmp. $137-141^{\circ} \mathrm{C}$, nach Umlösen aus Cyclohexan/Methylenchlorid gelbe Kristalle mit Schmp. $169-173^{\circ} \mathrm{C}$.

IR (KBr): $\mathrm{C}_{6} \mathrm{H}_{5}$ und $\mathrm{C}=\mathrm{N} 1589,1571,1537$; intensive Banden bei $693,699,746,761 \mathrm{~cm}^{-1}$ (u. a. $\mathrm{C}_{6} \mathrm{H}_{5}$-Wagging). - UV (Cyclohexan): $\lambda_{\max }$ (E) $302 \mathrm{~s}$ (7400), 314 (8400), 325s (7800), $339 \mathrm{~nm} \mathrm{~s}$

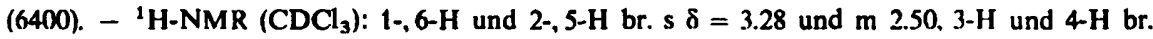

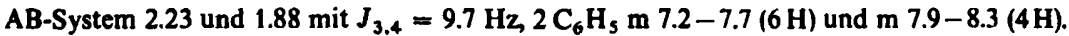

$$
\mathrm{C}_{20} \mathrm{H}_{16} \mathrm{~N}_{2} \text { (284.4) Ber. C } 84.48 \text { H } 5.67 \text { N } 9.85 \text { Gef. C } 84.18 \text { H } 5.37 \text { N } 10.02
$$

8,9-Diazatetracyclo/4.4.0.0 $2.4 .0^{3.5}$ /deca-7,9-dien-7,10-dicarbonsäure-dimethylester $(5 \mathrm{~b}): 1.00 \mathrm{~g}$ (5.05 mmol) 1,2,4,5-Tetrazin-3,6-dicarbonsäure-dimethylester ${ }^{33)}$ (3b) wurden bei Raumtemp. portionsweise mit einem kleinen Überschuß von etherischem 2 (ca. $5.10 \mathrm{mmol}$ in $14 \mathrm{ml}$ Ether) versetzt und solange gerührt, bis die roten Kristalle des Tetrazins verschwunden waren (15-30 min). Nach Absaugen und Waschen mit Ether erhielt man $1.10 \mathrm{~g}(87 \%) 5 \mathrm{~b}$ mit Schmp. $104-108^{\circ} \mathrm{C}$.

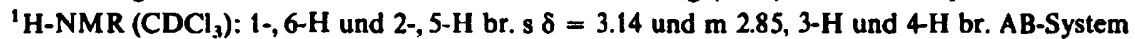
2.48 und 2.00 mit $J_{3,4}=9.5 \mathrm{~Hz}, 2 \mathrm{OCH}_{3}$ s 4.05 .

15,16-Diazaoctacyclo $\left[6.6 \cdot 2.0^{2.7} \cdot 0^{3.5} \cdot 0^{4.6} \cdot 0^{9.14} \cdot 0^{10.12} \cdot 0^{11.13}\right.$ ] hexadeca-15-en-1,8-dicarbonsäure-dimethylester (7): $9.90 \mathrm{~g}$ ( $50.0 \mathrm{mmol}) 3 \mathrm{~b}$ wurden bei Raumtemp. mit $250 \mathrm{ml}$ etherischem 2 (ca. $110 \mathrm{mmol}) 20 \mathrm{~h}$ gerührt. Die roten Tetrazinkristalle wichen dabei innerhalb von $4 \mathrm{~h}$ einer gelben Suspension (5b), die sich im weiteren Verlauf entfärbte. Am Ende der Reaktion wurde der farblose Niederschlag abgesaugt und mit Ether gewaschen: $12.82 \mathrm{~g} \mathrm{7,} \mathrm{nach} \mathrm{Umlösen} \mathrm{aus} \mathrm{Aceton}$ $11.31 \mathrm{~g}(69 \%) \mathrm{mit}$ Schmp. $211-212^{\circ} \mathrm{C}$ (Zers.)

IR $(\mathrm{KBr}): \mathrm{C}=\mathrm{O}$ 1732; weitere intensive Banden bei 1270, 1133, 1107 und $760 \mathrm{~cm}^{-1}$. - UV (Ethanol): $\lambda_{\max } 373 \mathrm{~nm}, \varepsilon$ 75. - ${ }^{1} \mathrm{H}-\mathrm{NMR}\left(\mathrm{CDCl}_{3}\right): 2-, 7-, 9-, 14-\mathrm{H}$ und 3-, 6-, 10, 13-H br. $\mathrm{s}$ $\delta=2.44$ und br. $\mathrm{s} 1.84,4,11-\mathrm{H}$ und 5-, $12-\mathrm{H} . \mathrm{m} 1.89$ und $\mathrm{m} 1.67 \mathrm{mit} J_{4.5} \approx 9.0 \mathrm{~Hz}, 2 \mathrm{OCH}_{3} \mathrm{~s}$ 3.96. - ${ }^{13} \mathrm{C}-\mathrm{NMR}\left(\mathrm{CD}_{2} \mathrm{Cl}_{2}\right)$ : s. Schema 1 .

$\mathrm{C}_{18} \mathrm{H}_{18} \mathrm{~N}_{2} \mathrm{O}_{4}$ (326.4) Ber. C 66.24 H $5.56 \mathrm{~N} 8.58$ Gef. C 66.04 H 5.70 N 8.88 Molmasse 327 (osmometr. in Benzol)

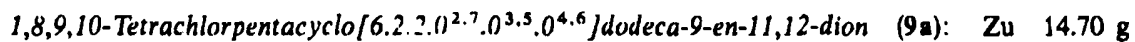
( $59.8 \mathrm{mmol}$ ) Tetrachlor-o-chinon (8a) tropfte man unter Kühlung mit Wasser und Rühren solange etherisches 2. bis der penetrante 2-Geruch bestehen blieb und die Farbe des Reaktionsgemisches von Rot nach Gelb umgeschlagen war; dies dauerte nur wenige Minuten. Nach Absaugen und Waschen mit Ether erhielt man $12.08 \mathrm{~g}$, aus der Mutterlauge weitere $0.68 \mathrm{~g} \mathrm{9a} \mathrm{(zusammen} 65 \%$ ) als gelbe Kristalle mit Schmp. $190-198^{\circ} \mathrm{C}$, nach Umfällen aus Methylenchlorid/Hexan 198 bis $200^{\circ} \mathrm{C}$. Die inverse Arbeitsweise, d. h. Zugabe von festem 8 a zum etherischen 2, lieferte $90 \%$ A usbeute.

IR (Nujol): C $=0$ 1742, $1762 \mathrm{~cm}^{-1}$. - UV (Cyclohexan): $\lambda_{\max }(\varepsilon) 445$ (130), $310 \mathrm{~s}$ (250), $290 \mathrm{~s}$ (770), $280 \mathrm{~nm} \mathrm{~s}(940)$. - MS (70 eV): $m / \mathrm{e} 326,324,322\left(0.06,0.11,0.09 \%, \mathrm{M}^{+}\right), 233$ (16), 231 (17), 198 (19), $196(30), 160(15), 78(44), 52\left(100, \mathrm{C}_{4} \mathrm{H}_{4}^{+}\right)$- ${ }^{1} \mathrm{H}-\mathrm{NMR}\left(\mathrm{CDCl}_{3}\right): 2-, 7-\mathrm{H}$ und 3-, 6-H br. $8 \delta=2.70$ und $\mathrm{m} 2.47,4-\mathrm{H}$ und $5-\mathrm{H} \mathrm{m} 2.23$ und $\mathrm{m} 2.45 \mathrm{mit} J_{4.5} \approx 9.2 \mathrm{~Hz} .-{ }^{13} \mathrm{C}-\mathrm{NMR}\left(\mathrm{CDCl}_{3}\right)$ : s. Schema 1.

$$
\mathrm{C}_{12} \mathrm{H}_{6} \mathrm{Cl}_{4} \mathrm{O}_{2}(324.0) \text { Ber. C } 44.49 \mathrm{H} 1.87 \mathrm{Cl} 43.77 \text { Gef. C } 44.32 \mathrm{H} 2.11 \mathrm{Cl} 44.18
$$

Pentacyclo $\left[6.2 .2 .0^{2.7}, 0^{3.5} .0^{4,6}\right.$ Jdodeca-9-en-11,12-dion $(9 \mathrm{~b}): 6.17 \mathrm{~g}(57.1 \mathrm{mmol}$ ) rohes 0 -Benzochinon ${ }^{34)}(8 \mathrm{~b})$ wurden mit $70 \mathrm{ml}$ etherischem 2 ( $58.0 \mathrm{mmol}$ ) versetzt und bei Raumtemp. gerührt. Es bildete sich eine dunkle Suspension. Das NMR-Spektrum der Lösung zeigte nach $5 \mathrm{~min}$

34) L. Horner und W. Dürckheimer, Z. Naturforsch., Teil B 14, 741 (1959); Farbwerke Hoechst AG (Erf. L. Horner und W. Dürckheimer), D.A.S. 1126852 (18. Okt. 1958) [C. A. 57, 8504 (1962)]. 
praktisch noch keinen Umsatz an, nach $3 \mathrm{~h}$ jedoch war die Reaktion zu mehr als der Hälfte abgelaufen. Man ließ weitere $15 \mathrm{~h}$ rühren und saugte dann $2.92 \mathrm{~g}$ eines grünbraunen Festkörpers ab, der laut NMR-Analyse $0.91 \mathrm{~g}$ reines $9 \mathrm{~b}$ enthielt. Das Filtrat wurde mit $20 \mathrm{ml} \mathrm{n}$-Hexan versetzt und auf $-70^{\circ} \mathrm{C}$ gekühlt. Dabei schieden sich $4.42 \mathrm{~g}$ gelbbraune Kristalle ab, die nach dem Waschen mit einem kalten 1 : 1-Ether/Hexan-Gemisch und Trocknen Schmp. $115-120^{\circ} \mathrm{C}$ zeigten. Aus dem Mutterlaugenriickstand wurden durch Destillation bei $80-100^{\circ} \mathrm{C}(\mathrm{Bad}) / 5 \cdot 10^{-5}$ Torr weitere $0.85 \mathrm{~g} 9 \mathrm{~b}$ als gelbe, rasch kristallisierende Flïssigkeit gewonnen. Ausb. $6.18 \mathrm{~g}(58 \%)$. Man nahm das Rohprodukt in absol. Ether auf, filtrierte und engte bei Raumtemp. soweit ein, daB sich die Hauptmenge in gelben Prismen mit Schmp. $118-121^{\circ} \mathrm{C}$ (Zers.) abschied. Die Substanz kann, allerdings verlustreich, bei $80-90^{\circ} \mathrm{C}(\mathrm{Bad}) / 5 \cdot 10^{-5}$ Torr sublimiert werden.

IR (KBr): $C=O$ 1723, $1739 \mathrm{~cm}^{-1}$. - UV (Cyclohexan): $\lambda_{\max }$ (E) 462 (93), $213 \mathrm{~nm} \mathrm{~s}$ (3400). MS (70 eV): $m / e 186\left(9 \%, M^{+}\right), 158\left(8, M^{+}-C O\right), 130\left(14, M^{+}-2 C O\right), 129(19), 128(8), 115$ (11), $52\left(100, \mathrm{C}_{4} \mathrm{H}_{4}^{+}\right)$. - ${ }^{2} \mathrm{H}-\mathrm{NMR}\left(\mathrm{CDCl}_{3}\right): 2-, 7-\mathrm{H}$ und 3-, 6-H br. $\mathrm{S} \delta=2.42$ und br. s $2.11,4-\mathrm{H}$ und 5- $\mathrm{H} \mathrm{m} 2.12$ und $1.77 \mathrm{mit} J_{4,5} \approx 9.0 \mathrm{~Hz}, 1-, 8-\mathrm{H} \mathrm{m} \mathrm{3.47,9-,} \mathrm{10-H} \mathrm{m} \mathrm{6.12.} \mathrm{-}{ }^{13} \mathrm{C}-\mathrm{NMR}\left(\mathrm{CDCl}_{3}\right)$ : s. Abb. 1 und Schema 1.

$$
\mathrm{C}_{12} \mathrm{H}_{10} \mathrm{O}_{2} \text { (186.2) Ber. C } 77.40 \text { H } 5.41 \text { Gef. C } 77.31 \text { H } 5.59
$$

Chinoxalin 10a aus 9a: $1.00 \mathrm{~g}(3.1 \mathrm{mmol}) 9 \mathrm{a}$ und $0.33 \mathrm{~g}(3.1 \mathrm{mmol})$ o-Phenylendiamin wurden in $50 \mathrm{ml}$ Benzol $1 \mathrm{~h}$ am Wasserabscheider unter Rückfluß gekocht. Nach dem Abkühlen filtrieste man von einem dunklen Niederschlag ab und engte das gelbe Filtrat i.Vak. ein. Der kristalline Rückstand lieferte nach Umlösen aus Methanol/Methylenchlorid $0.98 \mathrm{~g}(80 \%) 10 \mathrm{a}$ als fast farblose Kristalle mit Schmp. $220-226^{\circ} \mathrm{C}$; nach weiterem Umlösen Schmp. $225-229^{\circ} \mathrm{C}$.

MS (70 eV): $m / e$ 394, 396, $398\left(\mathrm{M}^{+}\right)$. - ${ }^{1} \mathrm{H}-\mathrm{NMR}\left(\mathrm{CDCl}_{3}\right): 2-, 7-\mathrm{H}$ und 3-, 6- $\mathrm{H}$ br. s $\delta=2.73$ und br. $\mathrm{s}$ 2.40, 4- $\mathrm{H}$ und 5- $\mathrm{H} \mathrm{m}$ 2.0-2.6, $\mathrm{C}_{6} \mathrm{H}_{4} \mathrm{AA}^{\prime} \mathrm{BB}^{\prime}$-System 7.6-8.0 und 8.0-8.4.

$$
\mathrm{C}_{18} \mathrm{H}_{10} \mathrm{Cl}_{4} \mathrm{~N}_{2} \text { (396.1) Ber. C } 54.58 \text { H } 2.54 \text { N } 7.07 \text { Gef. C } 54.70 \mathrm{H} 2.88 \text { N } 6.88
$$

Chinoxalin 10b aus $9 \mathrm{~b}$ : Nach der Vorschrift für $10 \mathrm{a}$ wurden aus $420 \mathrm{mg}(2.2 \mathrm{mmol}) 9 \mathrm{~b} 580 \mathrm{mg}$ $(100 \%)$ rohes und durch Umlösen aus Methanol $360 \mathrm{mg} \mathrm{(63 \% )} \mathrm{reines} 10 \mathrm{~b}$ als bräunliche Nadeln mit Schmp. $144^{\circ} \mathrm{C}$ erhalten.

MS (70 eV): $m / e 258\left(32 \%, M^{+}\right), 257(38), 193(39), 180\left(100, M^{+}-C_{6} \mathrm{H}_{6}\right), 78\left(35, \mathrm{C}_{6} \mathrm{H}_{6}^{+}\right)$. ${ }^{2} \mathrm{H}-\mathrm{NMR}\left(\mathrm{CDCl}_{3}\right): 2-, 7-\mathrm{H}$ und 3-, 6-H br. $\mathrm{s} \delta=2.36$ und br. $\mathrm{s} 2.06,4-\mathrm{H}$ und $5-\mathrm{H} \mathrm{m} 2.13$ und

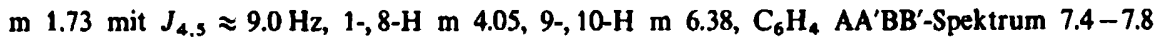
und 7.8-8.2.

$$
\mathrm{C}_{18} \mathrm{H}_{14} \mathrm{~N}_{2} \text { (258.3) Ber. C } 83.69 \text { H } 5.46 \mathrm{~N} 10.84 \text { Ger. C } 83.35 \text { H } 5.67 \mathrm{~N} 10.38
$$

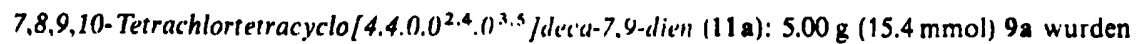
in $250 \mathrm{ml}$ Benzol in einem Pyrexgefäß durch einen 350-nm-Strahler mit einer Hg-Niederdrucklampe (Gräntzel-Reaktor 400 ) belichtet, bis die Gasentwicklung aufhörte ( $34 \mathrm{~h}$ ). Nach dem Abdampfen des Benzols binterblieben $4.10 \mathrm{~g}(99 \%)$ braunes kristallines $11 \mathrm{a}$, das durch Filtration seiner Hexanlösung durch $50 \mathrm{~g} \mathrm{Al}_{2} \mathrm{O}_{3}$ der Aktivitätsstufe III entfärbt wurde und nach Tieftemperaturkristallisation aus Hexan bei $85-87^{\circ} \mathrm{C}$ schmolz; Sdp. $80-90^{\circ} \mathrm{C}$ (Bad)/0.01 Torr.

IR (KBr): vC -H 3140, 3122, 3059, 2925; C =C $1635 \mathrm{~cm}^{-1}$. - UV (Ethanol): $\lambda_{\max }(\varepsilon) 306$ (4070), 292 (6670), 281 (6120), 270 (4560), 259 (3530), $240 \mathrm{~nm}$ (3870). - MS (70 eV): $m / e$ 270, 268, $266\left(1.3,2.7,2.4 \%, M^{+}\right) ; 233,232,231,230\left(1.8,1.6,1.8,2.1, M^{+}-C l\right) ; 218,216,214(1.1,2.1$, 1.6. $\left.\mathrm{C}_{6} \mathrm{H}_{2} \mathrm{Cl}_{4}^{+}\right): 198.196\left(6,11, \mathrm{M}^{+}-2 \mathrm{Cl}\right): 52\left(100, \mathrm{C}_{4} \mathrm{H}_{4}^{+}\right)$- ' $\mathrm{H}-\mathrm{NMR}\left(\mathrm{CDCl}_{3}\right):$ 1-, 6- $\mathrm{H}$ und 2 -, 5- $\mathrm{H} \mathrm{m} \delta=2.91$ und $\mathrm{m} 2.67,3-\mathrm{H}$ und $4-\mathrm{H} \mathrm{dtt} 2.17$ und dtt $2.00 \mathrm{mit} J_{3,4}=9.0 \mathrm{~Hz}, J_{2,3}$ und $J_{2,4}=1.6$ und $1.9 \mathrm{~Hz}$ sowie $J_{1,3}$ und $J_{1,4}=0.75$ und $1.15 \mathrm{~Hz}$, wobei die Kopplungskonstanten einem in $\left[\mathrm{D}_{8}\right.$ ] Toluol aufgenommenen 90-MHz-Spektrum entstammen. - ${ }^{13} \mathrm{C}-\mathrm{NMR}\left(\mathrm{CDCl}_{3}\right)$ : s. Schema 1.

$$
\mathrm{C}_{10} \mathrm{H}_{6} \mathrm{Cl}_{4} \text { (268.0) Ber. C } 44.82 \text { H } 2.27 \mathrm{Cl} 52.92 \text { Gef. C } 44.83 \text { H } 2.43 \mathrm{Cl} 53.42
$$


Tetracyclo[4.4.0.02,4.03.5]deca-7,9-dien (11 b): $1.34 \mathrm{~g}$ (7.2 mmol) 9b wurden in $250 \mathrm{ml}$ Benzol belichtet wie bei 11a beschrieben. Nach $55 \mathrm{~h}$ war die Gasentwicklung beendet. Einengen und Destillieren erbrachte $350 \mathrm{mg}(37 \%) 11 \mathrm{~b}$ als farblose Flüssigkeit mit Sdp. $71-73^{\circ} \mathrm{C} / 14 \mathrm{Torr}$.

IR (Film): C - H 3020, 2875, C=C 1588; weitere intensive Banden bei 1378, 1370, 1112, 1083, 953, 807, 770, 752, $676 \mathrm{~cm}^{-1}$. - UV (Cyclohexan): $\lambda_{\max }(\varepsilon) 283(2060), 272$ (3820), $262(3940)$, 259 (3030), 245 s (2050), $220 \mathrm{~nm}$ s (2630). - MS (70 eV): $\mathrm{m} / \mathrm{e} 130\left(19 \%, \mathrm{M}^{+}\right), 129(43), 128(30)$, 127 (14), 115 (23), 102 (5), 91 (8), 77 (11), 63 (8), 52 (100, $\left.\mathrm{C}_{4} \mathrm{H}_{4}^{+}\right)$. - ${ }^{1} \mathrm{H}-\mathrm{NMR}\left(\mathrm{CDCl}_{3}\right):$ 1-, 6-H

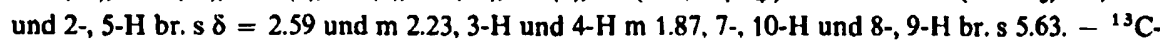
NMR $\left(\mathrm{CDCl}_{3}\right):$ s. Abb. 2 und Schema 1.

\section{$\mathrm{C}_{10} \mathrm{H}_{10}$ (130.2) Ber. C 92.26 H 7.74 Gef. C 91.42 H 8.03}

1,8,9,10,11,11-Hexachlorpentacyclo/6.2.1.0 2.7. $0^{3.5} \cdot 0^{4.6} /$ unlec-9-en (16a): $32.0 \mathrm{~g}$ (117 mmol) Hexachlorcyclopentadien (15) wurden in $210 \mathrm{ml}$ etherischem $2(111 \mathrm{mmol})$ aufgelöst. Nach $22 \mathrm{~h}$ bei Raumtemp. zeigte ein NMR-Spektrum der Lösung den vollständigen Umsatz des Benzvalens an. Man engte im Rotationsverdampfer ein. löste den Rückstand in möglichst wenig n-Hexan und kühlte auf $-70^{\circ} \mathrm{C}$. Die abgeschiedenen, farblosen Kristalle wurden abgesaugt, mit kaltem Hexan gewaschen und getrocknet: $26.0 \mathrm{~g} \mathrm{16a}$ mit Schmp. $120-123^{\circ} \mathrm{C}$ bei raschem Aufheizen. Aus dem Mutterlaugenrückstand destillierte bei $85-90^{\circ} \mathrm{C}(\mathrm{Bad}) / 0.01$ Torr überschüssiges $15 \mathrm{ab}$, während weiteres $16 \mathrm{a}$ bei $100-125^{\circ} \mathrm{C}(\mathrm{Bad}) / 0.01$ Torr überging, das nach Umlösen $2.0 \mathrm{~g}$ wog. Gesamtausb. $28 \mathrm{~g}(72 \%)$.

IR (KBr): C =C $1603 \mathrm{~cm}^{-1}$. - MS $(20 \mathrm{eV}): \mathrm{m} / \mathrm{e} 352,350\left(2.9,3.0 \%, \mathrm{M}^{+}\right), 315$ (30), 279 (52), 277 (35), 239 (30), 237 (23), $100(100), 52\left(100, \mathrm{C}_{4} \mathrm{H}_{4}^{+}\right)$. $-{ }^{1} \mathrm{H}-\mathrm{NMR}\left(\mathrm{CCl}_{4}\right)$ : br. s $\delta=3.00(2 \mathrm{H})$, m $2.1-2.3(4 \mathrm{H})$

$$
\mathrm{C}_{11} \mathrm{H}_{6} \mathrm{Cl}_{6} \text { (350.9) Ber. C } 37.65 \mathrm{H} 1.71 \text { Gef. C } 37.84 \mathrm{H} 1.75
$$

Pentacyclo/6.2.1.0 $0^{2.7} \cdot 0^{3.5} \cdot 0^{4.6}$ Jundec-9-en (16 b): Zu $11.5 \mathrm{~g}(500 \mathrm{mmol})$ Natrium kondensierte man $170 \mathrm{ml}$ flüssiges Ammoniak, tropfte dann unter Rühren bei $-70^{\circ} \mathrm{C} 12.0 \mathrm{~g}(34.2 \mathrm{mmol}) 16 \mathrm{a}$ in $50 \mathrm{ml}$ absol. Ether innerhalb von $30 \mathrm{~min} z \mathrm{zu}$, rührte $2 \mathrm{~h}$ bei $-70^{\circ} \mathrm{C}$ und neutralisierte bei $-70^{\circ} \mathrm{C}$ mit $26.8 \mathrm{~g}$ (500 mmol) Ammoniumchlorid. Man ließ auf Raumtemp. kommen, wobei das Ammoniak verdampfte, nahm den Rückstand mit Pentan und Wasser auf, trennte die Pentanphase ab, trocknete über $\mathrm{CaCl}_{2}$ und engte i. Vak. ein. Bei $69-70^{\circ} \mathrm{C} / 12$ Torr destillierten dann $1.53 \mathrm{~g}(31 \%)$ $16 \mathrm{~b}$ als farblose Flüssigkejt.

IR (Film): C-H 3025, 2960, 2925, 2865; weitere intensive Banden bei 1448, 1393, 1340, 1313 , 1295, 1253, 1235, 1228, 1107, 927, 912, 905, 847, 791, 757, $719 \mathrm{~cm}^{-1}$. MS $(20 \mathrm{eV}): \mathrm{m} / \mathrm{e} 144$ $\left(17 \%, \mathrm{M}^{+}\right), 143(11), 129(36), 79(72), 78\left(70, \mathrm{C}_{6} \mathrm{H}_{6}^{+}\right), 66\left(100, \mathrm{C}_{5} \mathrm{H}_{6}^{+}\right) .-{ }^{1} \mathrm{H}-\mathrm{NMR}\left(\mathrm{C}_{6} \mathrm{D}_{6}\right): 2-$, 7-H und 3-, 6-H br. $\mathrm{s} \delta=2.39$ und br. $\mathrm{s} 1.75,4-\mathrm{H}$ und 5-H $\mathrm{m} \mathrm{1.5-2.0,1-,} \mathrm{8-H} \mathrm{m} \mathrm{2.59,9-,} \mathrm{10-H} \mathrm{m}$ $5.89,11 \mathrm{a}-\mathrm{H} \mathrm{m} 1.36,11 \mathrm{~b}-\mathrm{H} \mathrm{m} 1.60, J_{1,11 \mathrm{a}}=1.6 \mathrm{~Hz}, J_{1,11 \mathrm{~b}}=1.9 \mathrm{~Hz}, J_{9.11 \mathrm{a}}=0.8 \mathrm{~Hz}, J_{11 \mathrm{a}, 11 \mathrm{~b}}=$ $8.0 \mathrm{~Hz}$ - ${ }^{13} \mathrm{C}-\mathrm{NMR}\left(\mathrm{C}_{6} \mathrm{D}_{6}\right):$ s. Schema 1 .

$$
\mathrm{C}_{11} \mathrm{H}_{12} \text { (144.2) Ber. C } 91.61 \text { H } 8.39 \text { Gef. C } 92.02 \text { H } 8.36
$$

